\title{
Iterative springback compensation of NUMISHEET benchmark \#1
}

\author{
R.A. Lingbeek ${ }^{* \dagger}$, J. Huétink ${ }^{* *}$, S. Ohnimus ${ }^{\dagger}$ and J. Weiher ${ }^{\dagger}$ \\ *Netherlands Institute for Metals Research, Rotterdamseweg137, 2628 AL Delft, The Netherlands \\ ${ }^{\dagger}$ INPRO Innovationsgesellschaft für fortgeschrittene Produktionssysteme in der Fahrzeugindustrie mbH \\ Hallerstraße 1, D-10587 Berlin \\ ${ }^{* *}$ University of Twente, Faculty of Engineering Technology, P.O. Box 217, 7500 AE Enschede, The Netherlands
}

\begin{abstract}
Upon unloading after the forming stage, a sheet metal product will spring back due to internal stresses. Springback is a major problem for process-planning engineers. In industrial practise, deformations due to springback are compensated manually, by doing extensive measurements on prototype parts, and altering the tool geometry by hand. This is a time consuming and costly operation. In this paper the application of two compensation algorithms, based on the finite element simulation of the forming process are discussed. The smooth displacement adjustment (SDA) method and the springforward (SF) method have been applied to several industrial products, such as the NUMISHEET 2005 benchmark\#1. With the SDA method successful compensations have been carried out. For the SF method some principal problems remain.
\end{abstract}

Keywords: Sheet metal forming, Springback, Compensation

PACS: <81.20.Hy $>$

\section{INTRODUCTION}

Compensating springback during tool design is one of the greatest challenges for the process engineers. Currently this is a process of trial and error. First, the product shape deviation is measured. Then a manual tool redesign is carried out. The prototype tools have to be reworked and another prototype part is produced. If the geometry is still not satisfactory more tool redesigns have to be carried out until the shape deviation is reduced sufficiently. Obviously, this is a time consuming and costly process.

Now that the simulation of the deep drawing process and the calculation of springback have become possible, the opportunity arises to compensate the toolset using simulation data. Because the geometries of the simulated FE blank-mesh can be evaluated in more detail the compensation can now be more accurate. Also, the cost and duration of FE simulations are much lower than the production of a prototype part, so considerably more tool-geometries can be evaluated in a short time.

The two most effective algorithms for springback compensation are called 'Displacement Adjustment' or DAmethod and the 'Spring Forward' or SF-method [1]. The DA-method [2] [3] performed very well on some academic example products, but for industrial products some practical issues had to be solved. This resulted in the Smooth Displacement Adjustment or SDA-method [4].

\section{THE SDA METHOD}

The DA compensation is directly based on the springback displacement. Regard the reference or desired geometry $\mathbf{R}$, given as a collection of $n$ points in $\Re^{3}$ and the springback geometry $\mathbf{S}$

$$
\begin{aligned}
& \mathbf{R}=\left\{\mathbf{r}_{i} \mid \mathbf{r}_{i} \in \mathfrak{R}^{3}\right\}, 0<i<n \\
& \mathbf{S}=\left\{\mathbf{s}_{i} \mid \mathbf{s}_{i} \in \mathfrak{R}^{3}\right\}, 0<i<n
\end{aligned}
$$

The compensated geometry $\mathbf{C}$ is now calculated as follows:

$$
\mathbf{C}=\mathbf{R}+a(\mathbf{S}-\mathbf{R})
$$

The factor $a$ is the overbending factor. It is generally negative and in practise it's value varies between -2.5 and -0.6 , depending on the product geometry and forming process. $a(\mathbf{S}-\mathbf{R})$ is called the shape modification field $\Phi$. The DA method can also be applied iteratively. The first compensated geometry $\mathbf{C}$ is now referred to as $\mathbf{C}^{1}$ , and with this geometry a new FE simulation is carried out. The resulting springback mesh $\mathbf{S}^{1}$ is now used to modify $\mathbf{C}^{1}$, delivering the second compensated geometry $\mathbf{C}^{2}$. Note that $\mathbf{R}$ and $\mathbf{S}^{1}$ are the results from different FE simulations. The iterative formulation of equation 3 in iteration $j$ is:

$$
\mathbf{C}^{j+1}=\mathbf{C}^{j}+a\left(\mathbf{S}^{j}-\mathbf{R}\right)
$$

The advantage of iterative application is that the process engineer does not need to guess an overbending factor because the tool geometry converges to it's optimal 
shape iteratively. Another advantage is that, depending on the product's geometry and forming process, the accuracy can also be raised. The factor $a$ is still present in the formula to gain control over the amount of compensation that is applied per iteration. A value of 1.0 is recommended. However, in some cases this leads to large changes in shape of the tool geometry. This may cause the springback behavior of the forming process to change, resulting in convergence problems. In this case is recommended to start with a lower $a$-value.

The compensation field $\Phi=a\left(\mathbf{S}^{j}-\mathbf{R}\right)$ is only known on the nodes of the reference mesh $\mathbf{R}$. This means that only the product itself can be compensated. To be able to apply the shape modification field $\Phi$ to any mesh, including the generally larger and topologically different tool meshes, or even the analytically defined CAD geometries, the discrete field needs to be approximated and extrapolated by an analytical function $\Psi$. With this addition, the method is now called the Smooth Displacement Adjustment (SDA) method [4].

The approximation function needs to be very flexible in order to capture small shape deviations, but it also needs to remain stable so no unwanted waviness is introduced in the compensated geometry. To achieve this, a trivariate $\mathrm{B}$-spline volume is used:

$$
\Psi(x, y, z)=\sum_{i} \sum_{j} \sum_{k} N_{i, p}(x) N_{j, p}(y) N_{k, p}(z) \mathbf{P}_{i, j, k}
$$

where $\mathbf{P}_{i, j, k}$ is a three dimensional array with so-called 'control points', and $N_{i, p}$ a B-spline basis function. Least squares fitting is used to find the optimal set of control points in with the following equation:

$$
|\Phi-\Psi(x, y, z)|_{L 2} \rightarrow \min
$$

Now geometries with arbitrary topology can be compensated in the same way as equation 3:

$$
\mathbf{C}^{j+1}=\mathbf{C}^{j}+\Psi^{j}\left(\mathbf{C}^{j}\right)
$$

For a detailed discussion on the calculation of the approximation function the reader is referred to [4].

\section{COMPENSATING THE NUMISHEET BENCHMARK PRODUCT}

In various publications, springback compensation was carried out for relatively simple products. The main focus is the hat-profile, which is basically an elastoplastic 2D bending problem. In reality, springback depends not only on bending stresses but also on in-plane stresses. This is

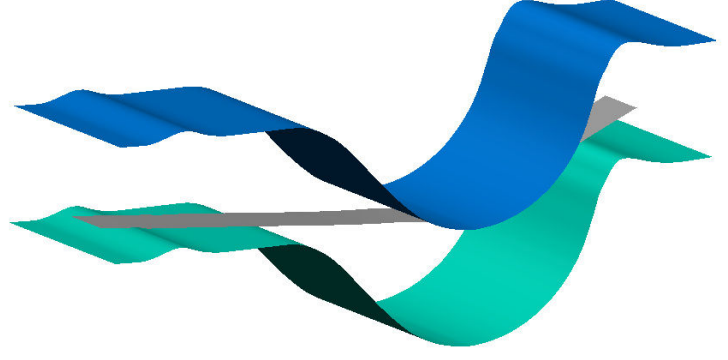

Figure 1. The forming process in PAM-STAMP

important for the compensation. Since the tool geometry is changed, and not the geometry of the product itself, the result of the compensation also depends on the behavior of the deep drawing process with respect to small changes in the tool geometry. For 2D bending problems this response is mainly linear and the results of the (S)DA algorithm are very good.

\section{A free bending product}

The following example process consists of the free bending of a strip. The process is a simplification of a real industrial process, and is shown in figure 1 . The process has been modeled in PAM-STAMP 2G 2004. The forming process was calculated with an explicit solver, for the springback phase the implicit solver was used.

The SDA code was implemented in $\mathrm{C}++$. The program is able to set-up and start PAM-STAMP simulations and to evaluate the results of the simulation and compensate the tool meshes, no user interaction is required. During the iterations, an overbending factor of -1.0 was used. The results of an iterative compensation are shown in the graph in figure 2 . The shape deviation between the reference or desired geometry and the actual produced geometry is lowered by $96 \%$ (regarding the maximum shape deviation).

Interestingly, the amount of compensation varies over the product. In one compensation iteration the overbending factor $a$ is the factor between the springback displacement and the compensation displacement. This can be generalized to a 'local overbending factor' $a_{i}$ at node $i$ :

$$
a_{i}=\frac{\left|\mathbf{c}_{i}-\mathbf{r}_{i}\right|}{\left|\mathbf{s}_{i}-\mathbf{r}_{i}\right|}
$$

Note that for a one-step iteration this value is of course identical for each node. In the middle of the blank, $a_{i}$ is 


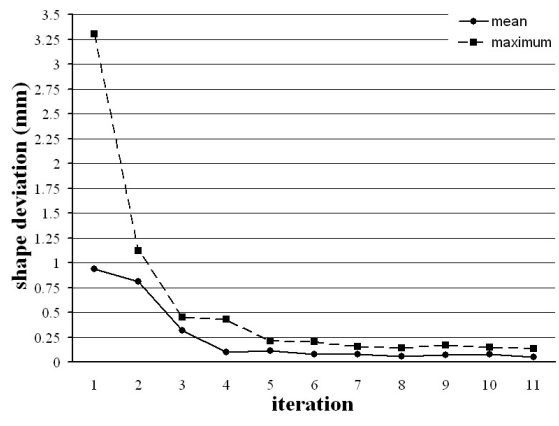

Figure 2. Shape deviation during compensation

about -1.30 (the industry rule-of-thumb), at the sides it is much lower, around -0.60 .

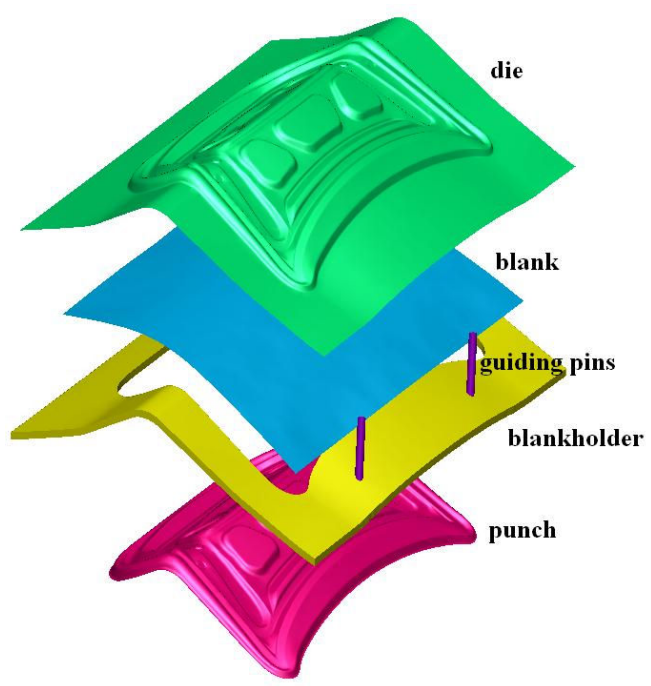

Figure 3. The forming process of the benchmark part

\section{The NUMISHEET benchmark}

The NUMISHEET benchmark part, a trunk-lid inner frame, is produced with a deep drawing process followed by a trimming operation. As shown in picture 3, a pre-formed blank was used. Due to the complexity of the springback shape deviation, a very flexible approximation function was used, using a control grid of 5 by 5 by 5 control points( 375 parameters). The more flexible the spline function gets, the less stable it becomes for extrapolation. In practise this means that outside the product area of the tools the compensation function tends to behave badly and the tool shape gets damaged, as shown in figure 4.

Therefore a cut-off function $K$ has been developed. The idea of this function is to multiply the compensation
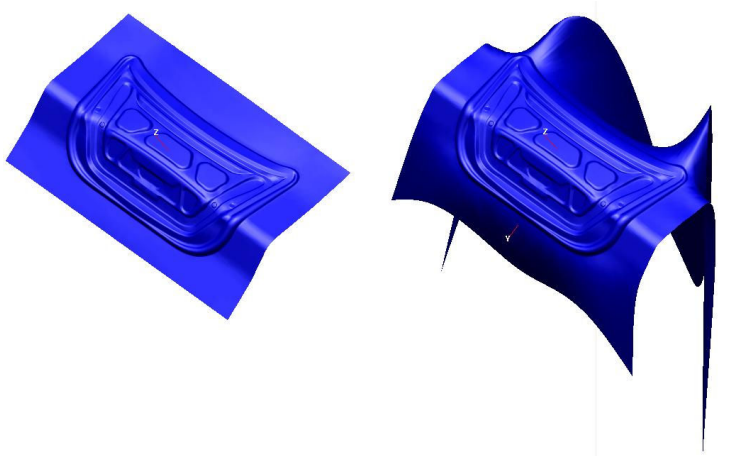

Figure 4. Extrapolation problems for the die

vector for each tool node $\mathbf{c}_{i}$ with a so-called cutoff value $k_{i}$ between 0.0 and 1.0 .

$$
\mathbf{c}_{i}^{j+1}=\mathbf{c}_{i}^{j}+k_{i} \Psi^{j}\left(\mathbf{c}_{i}^{j}\right)
$$

If the tool-node that is to be compensated is in the product area, the cutoff value is 1.0. If it is in the blankholder area, the value becomes 0.0. Between the two areas the function value drops smoothly. In this way, detailed compensation is possible, while leaving the blankholder area unchanged.

Another problem is refinement. To enhance the speed and accuracy of the simulation, refinement was used during the simulation. This presents a problem for equation 4 . Because of the changes in the tool geometry the refinement changes slightly during different iterations, so for each iteration the topology of the springback mesh is slightly different. It also varies because of small changes in the trimming process. An algorithm was developed to remove the refined elements from the springback meshes and make them topologically identical to the reference mesh.

\section{RESULTS}

Due to the more complex forming process, an overbending factor of 0.3 was chosen during the consecutive iterations, in order to keep changes to the process and the springback deformation at minimum. An approximation function with 125 parameters was used. The shape deviation before and after compensation is pictured in figure 8. In most parts of the product, the shape deviation has been lowered significantly. During the compensation, the smoothness of the tool surface was also preserved, due to the smoothing of the approximation surface (see figure 6, courtesy of ICEM software). The results are also plotted as the grey lines in figure 5. The mean shape deviation calculated as follows: 


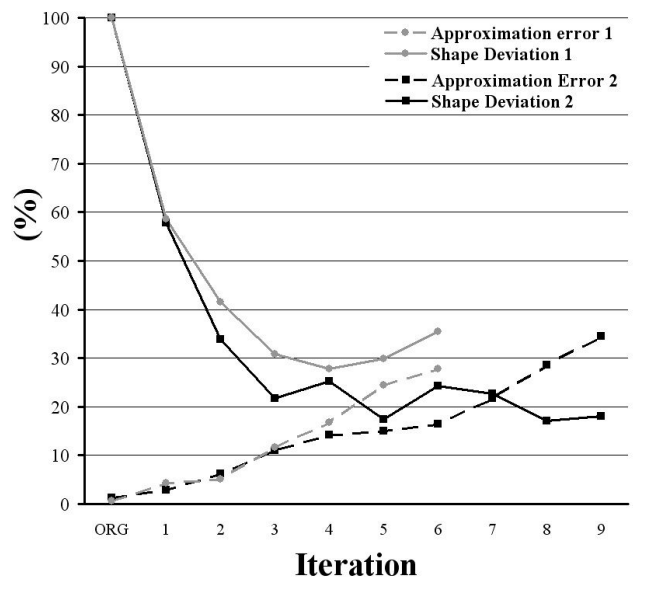

Figure 5. Results for the compensation of the benchmark part

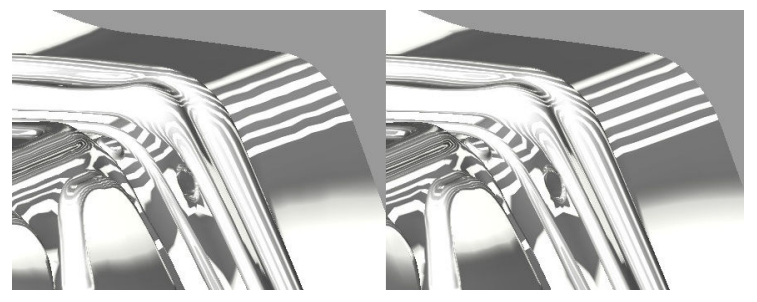

Figure 6. Tool light reflection before (left) and after compensation (right). Image courtesy of ICEM software

$$
\varepsilon_{\text {geom }}=\frac{\sum_{i}\left|\mathbf{r}_{i}-\mathbf{s}_{i}^{j}\right|^{2} A_{i}}{\sum_{i}\left|\mathbf{r}_{i}-\mathbf{s}_{i}^{0}\right|^{2} A_{i}}
$$

The 'nodal surface' $A_{i}$ (see figure 11) used for weighing is the so-called Voronoi area that surrounds a node in the mesh. A description can be found in [5]. The mean shape deviation is reduced by around $70 \%$, a good results but significantly worse than the simple bending product.

One of the reasons for this are the limitations of the approximation function, as can be concluded from the grey dashed graph. This graph displays the approximation error of the approximation function. In this figure $\mathbf{u}$ is the shape deviation vector, and $\mathbf{u}_{a p p}$ is the approximated shape deviation.

$$
\varepsilon_{\text {app }}=\frac{\sum_{i}|\mathbf{e}|^{2} A_{i}}{\sum_{i}|\mathbf{u}|^{2} A_{i}}
$$

After the fifth iteration, this error has risen to over $25 \%$. This indicates that the 'wavelength' of the remaining shape deviation has become so small that it cannot be captured effectively anymore by the approximation

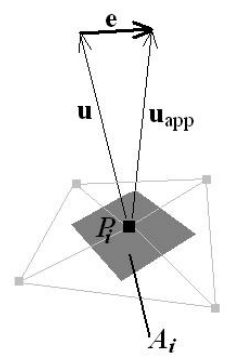

Figure 7. Mean approximation error calculation.

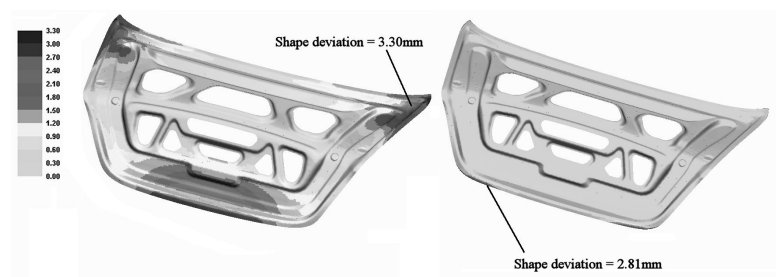

Figure 8. Shape deviation with original tools (left) and compensated tools (right)

function. Note that the shape deviation does not need to be small. This is shown in figure 8; at the flange of the product a large local shape error remains.

To verify this theory, another compensation process has been carried out, this time using a spline volume function with 7 by 7 by 7 control points was used, with a total of 1029 parameters. This function turned out to be so unstable that even with the cut-off function, the die geometry would become damaged in the die-addendum area already. As a solution, fictive points with zero shape deviation were added to the shape deviation field, to stabilize the function outside the product area. This is explained in figure 9.

The results of a compensation with this function are also shown in figure 5 and represented by the black lines. Now a reduction in shape deviation of around $80 \%$ (regarding the mean shape deviation) was achieved. The approximation error is significantly lower than with the previous settings, but it also rises rapidly during the

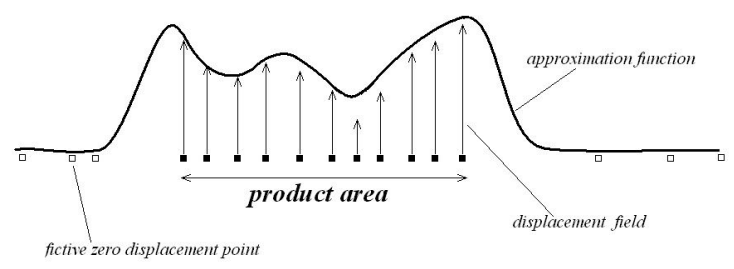

Figure 9. Stabilizing the approximation function 


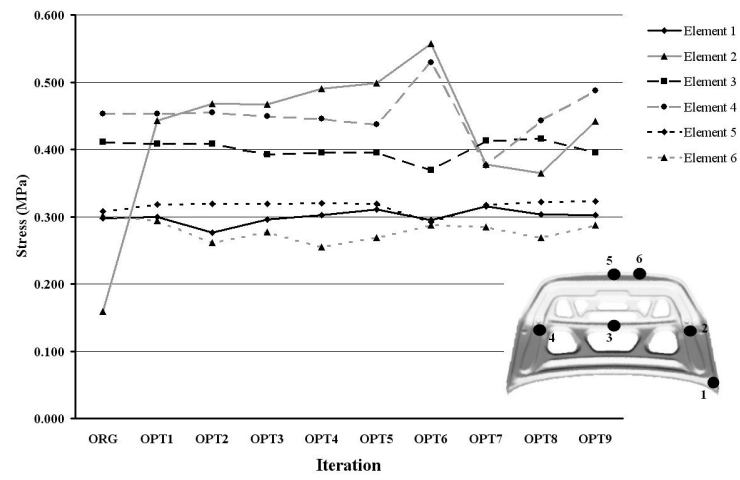

Figure 10. equivalent membrane stress during compensation

iterations.

The question is now, why is the result so much better for the free-bending problem? A first hypothesis is that even though a low compensation factor was used during the iterations, the process changes too much and convergence becomes worse. In the graph in figure 10, the equivalent membrane stress after forming but before springback at various locations in the blank is visualized. A variation of around $15 \%$ is found for all points except point 2 and 4. This is not surprising since at those locations heavy wrinkling occurs during the deep drawing process. The same analysis has been carried out for the strip bending process. Here the variation in stress at various points in the blank is also around $15 \%$.

Another way to look at process stability is to compare the springback displacement at various locations in the blank. Again, both processes show less than $10 \%$ variation during the iterations. So, it can be concluded that process instability is not the cause for the lower efficiency of the compensation on the benchmark part.

\section{THE PBSF ALGORITHM}

The spring forward (SF) method was introduced by Karafillis and Boyce in [6]. The principle of the SF method is to compensate springback with the internal stresses that cause it, instead of applying straightforward geometric optimization, such as the DA method. The SF method consists of four steps. In step 1, the forming process is simulated. After the forming of the product, the contact forces of the tools acting on the product are 'measured' from the FE result files (step 2). As a compensation measure, this force-field $f$ is reversed and applied to the (stress free) product geometry in a separate FE calculation in step 3. The idea behind this is that when the tools are closed the blank retains its shape due to the contact forces $(-f)$ of the tools. When the tools are removed, it is assumed that the blank springs back under the action of the 'internal force-field' $f$. So, by applying the force-field $-f$ to the reference geometry to produce the compensated geometry, it is assumed that the deformation due to springback is compensated. Finally, the obtained part shape is used to create new tools in step 4. Another iteration can be carried out by starting again at step 1 . Note that during the consecutive iterations, always the original part geometry is compensated with the force field.

Compared to the (iterative) DA algorithm the SF algorithm "converges more slowly, if at all, or may converge to incorrect die shapes" [1]. Even when the basic assumption of the method is correct, there is no fixed geometrical target, which is a possible cause for the convergence problems. This has been demonstrated using the plastic stretching of a bar as a model for the deep drawing process in [7]. For this process, the SF and DA methods have been carried out. The SF method converges slower than the DA method, and a shape error remains.

A solution for the above mentioned problem is the introduction of another step in a SF iteration: the pushback stage. Instead of using the tool-contact forces as the force field $f$, the springback mesh is pushed back into the right shape first and the (nodal) forces that are required are now taken as the force field $f$. With this push-back force-field the product is compensated. Another important change is that, in the second and following iterations the compensation is carried out on the compensated geometry of the previous iteration.

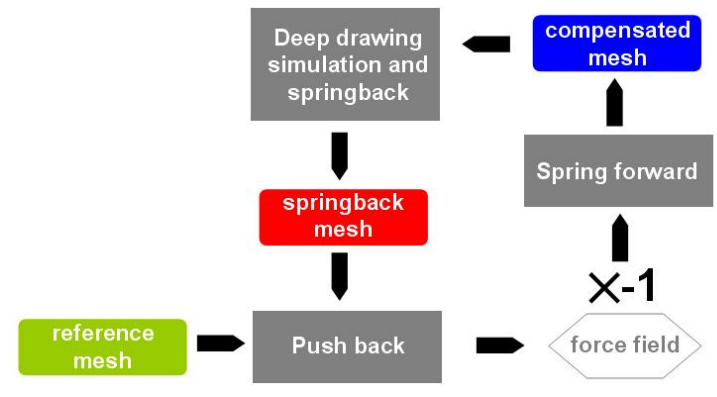

Figure 11. Principle of the PBSF method

So, in the second and following iterations, the springback geometry is much closer to the reference geometry. This means that the push back forces become lower with each iteration. With this assumption, the spring forward forces are linked to the reference geometry, and convergence is much more likely. In this form the method is now called Push-back Spring-Forward or 
PBSF. The method has been successfully applied to a 2D strip elastoplastic bending problem.

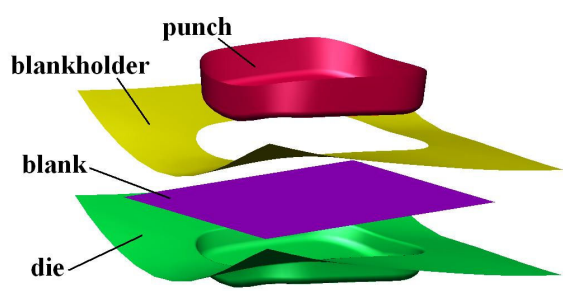

Figure 12. The deep drawing process for the fuel tank cap

The PBSF principle is now tested with an industrial part, a fuel tank cap. Its production comprises a forming and a trimming stage. For demonstrating the algorithm, only the forming step, shown in picture 12 is taken into consideration. The deep drawing stage was calculated with ABAQUS/explicit, the springback stage with the implicit code ABAQUS/standard. During springback (and the push-back and springforward calculations) the blank was fixed statically in the middle.

Firstly the blank was pushed back into it's desired shape at all nodes in the mesh. Note that this is not principally necessary, a smaller number of push-back points can also be selected. However, then the shape of the blank will not exactly reflect the desired shape during push-back. The push-back force-field was reversed and applied to the blank in an implicit ABAQUS calculation. The load was not applied at once, but a smooth load-magnitude curve was used. Unfortunately the calculation diverges at approximately half the load, and the springforward shape cannot be calculated. The reason can be found in the stress-diagrams shown in picture 13.

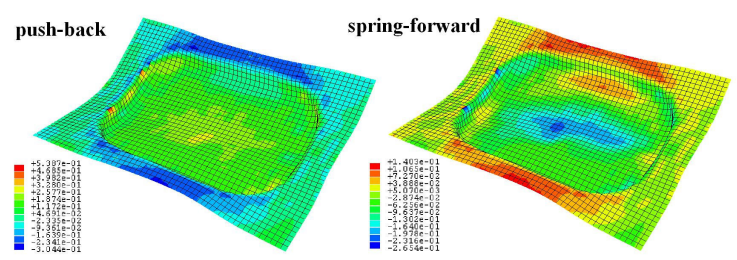

Figure 13. In-plane stress $\sigma_{11}$ in the push-back and springforward calculations

As expected, the stress-field in the flange of the springforward blank reflects the stress-field in the push-back blank, but the sign has changed and the value is about $50 \%$ lower (because the calculation was terminated halfway). Around the fixing point, in in the middle of the blank, large compressive in-plane stresses $\sigma_{11}$ and $\sigma_{22}$ (not pictured) are observed. This leads to local buckling and is the cause for the abrupt divergence in the FE calculation.

When less push-back points are used, the buckling problem during the spring-forward calculation is avoided. Unfortunately, the blank now starts to rotate around it's fixing point. This effect is also mentioned in [2] as large unbalanced spring forward forces. During push-back, the blank is exactly in equilibrium. Then the forces from the deformed state are taken, reversed and applied to the undeformed state. Since springback is a geometrically non-linear process, the geometrical difference between both states cannot be neglected. So after application of the springforward forces, a large internal imbalance is caused in the blank resulting in heavy rotation. In [2] it has been reported that the convergence of the SF method depends heavily on the choice of the fixation point, this is due to the same reason.

\section{CONCLUSION}

The (S)DA method has again shown it's usefulness. Also on complex products, such as the NUMISHEET 2005 benchmark, good results can be achieved. The mean shape deviation of the product was lowered by $80 \%$ when using the compensated tools. However, some rather large local shape errors remain. For the (PB)SF method some complicated principal problems remain. Currently the application of the method is limited to $2 \mathrm{D}$ bending problems, in more complicated geometries the calculation of the springforward shape is not yet accomplished.

\section{REFERENCES}

1. R. Wagoner, "Fundamental aspects of springback in sheet metal forming," in proceedings ESAFORM, edited by V. Brucato, 2003, pp. 7-14.

2. W. Gan, and R. H. Wagoner, International Journal of Mechanical Science, 46, 1097-1113 (2004).

3. W. Gan, R. Wagoner, K. Mao, S. Price, and F. Rasouli, Journal of Material Processing and Technology, 126, 360-367 (2004).

4. R. Lingbeek, H. Huétink, S. Ohnimus, M. Petzoldt, and J. Weiher, The development of a finite elements based springback compensation tool for sheet metal products (2004), submitted to the Journal of Engineering Materials and Technology.

5. R. V. Garimella, and B. K. Swartz, Curvature estimation for unstructured triangulations of surfaces, Tech. Rep. LA-UR-03-8240, Los Alamos National Laboratory (2003).

6. A. Karafillis, and M. Boyce, Int. J. Mach. Tools. Manuf., 36, 503 (1996).

7. R. Lingbeek, Aspects of a designtool for springback compensation, Master's thesis, University of Twente (2003). 